\title{
Brain Abscess in a Patient with Osteopetrosis: A Rare Complication
}

\author{
(D) Merve İşeri Nepesov1, (D) Eylem Kıral2, (D) Gürkan Bozan², (D) Ömer Kılıç1', (D) Kürşat Bora Çarman³, (D) Coşkun Yarar33, \\ (1) Suzan Şaylısoy4, (1) Ener Çağrı Dinleyici² \\ 1Eskişehir Osmangazi University Faculty of Medicine, Department of Pediatric Infectious Diseases, Eskişehir, Turkey \\ 2Eskişehir Osmangazi University Faculty of Medicine, Department of Pediatric Intensive Care Unit, Eskişehir, Turkey \\ ${ }^{3}$ Eskişehir Osmangazi University Faculty of Medicine, Department of Pediatric Neurology, Eskişehir, Turkey \\ 4Eskişehir Osmangazi University Faculty of Medicine, Department of Radiology, Eskişehir, Turkey
}

\section{What is already known on this topic?}

The most common infectious complication of osteopetrosis is osteomyelitis; in particular, mandibular osteomyelitis may be seen due to bone sclerosis and reduced vascular supply. Otolaryngological complications, such as recurrent otitis media, are also seen frequently.

\section{What this study adds?}

To our knowledge, this report is only the second published description of brain abscess in association with osteopetrosis. Therefore, routine otologic examination should be an integral component of management and plays an important role in preventing more severe complications, such as brain abscess.

\section{Abstract}

Brain abscess formation is extremely rare in patients with osteopetrosis. Herein, we report a case of viridans streptococci brain abscess in an immunocompromised child diagnosed with osteopetrosis. The patient presented with a sudden change in mental status and convulsions. Radiological evaluation revealed a temporal lobe brain abscess, and intravenous antibiotherapy was started immediately. The patient underwent abscess drainage, and laboratory investigation of pus material revealed viridans streptococci.

Keywords: Osteopetrosis, chronic otitis media, brain abscess, viridans streptococci

\section{Introduction}

Osteopetrosis describes a group of rare genetic skeletal disorders characterized by reduced osteoclast activity, which results in defective bone resorption and increased bone mass and density (1). Osteopetrosis is normally classified descriptively by its clinical severity and inheritance pattern $(2,3)$. The adult (autosomal dominant) type is a mild form of the disease characterized by normal life expectancy while the infantile (autosomal recessive) type is characterized by diagnosis in early life and fatal prognosis. The intermediate type of osteopetrosis, which is a subgroup of the autosomal recessive type, is seen less frequently; diagnosis is made during early childhood, with patients being clinically normal at birth $(2,3,4)$. Currently, with the improvement in molecular and genetic techniques, forms of osteopetrosis can also be classified according to their genetic basis $(2,3)$. In autosomal recessive osteopetrosis, loss of bone marrow causes anemia, thrombocytopenia, and leukopenia, in turn resulting in extramedullary hematopoiesis, hepatosplenomegaly, and recurrent infection. Bone fractures with minor trauma and osteomyelitis of especially the long bones and mandible can be seen (1). Differential diagnosis of osteopetrosis including dysosteosclerosis, which is more rare, and is distinguished from osteopetrosis by the presence of early acquired 
sclerotic platyspondyly and metaphyseal expansion. Another differential diagnostic tool is mutation analysis (5).

The factors associated most strongly with the appearance of infectious complications in osteopetrosis are impaired resistance to infection due to neutropenia and reduced vascular supply to the bone, which limits the availability of antibiotics in the infected area. Otolaryngological complications, such as recurrent otitis media, are also seen frequently, but brain abscess formation due to recurrent otitis media is extremely rare. Herein, we discuss a case of viridans streptococci brain abscess in an immunocompromised child with osteopetrosis. To our knowledge, this report is only the second published description of brain abscess in association with osteopetrosis (6).

\section{Case Report}

A 14-year-old boy with a previous diagnosis of autosomal recessive osteopetrosis type 7 (OPTB7) presented to our pediatric emergency care unit with complaints of confusion, sudden abnormal involuntary muscle contractions, and temporary cessation of breathing and cyanosis. He had experienced nausea, vomiting, and diarrhea for the past two weeks. There was no history of trauma or fever. The patient had taken oral antibiotics, primarily amoxicillin/ clavulanic acid, on numerous occasions in the past year for recurrent suppurative otitis media. He had a history of frequent purulent otorrhea, which had never fully resolved. In his medical history, abnormal eye movement was first observed by his family when he was two months old and then, at age eight months, a bone fracture was seen. During follow-up, the patient was diagnosed with blindness caused by optic nerve compression and hypogammaglobulinemia in addition to multiple arm and leg fractures. He was diagnosed with osteopetrosis in the infancy period and a homozygous tumor necrosis factor receptor superfamily member $11 \mathrm{~A}$ (TNFRSF11A) mutation [c.838G > T (p.G280X)] was detected in an another tertiary hospital (7). There is parental consanguinity and both parents were heterozygous for the mutation. He was receiving intravenous immunoglobulin replacement therapy alone, with no other treatment.

On physical examination, the patient showed a change in mental status and abnormal involuntary contractions of the left arm. The Glasgow Coma Scale (GCS) score was 8 (E 2, $\mathrm{V} 2, \mathrm{M}$ 4). Intravenous midazolam was started immediately to control seizures. Laboratory examination revealed a white blood cell count of $17,500 / \mathrm{mm}^{3}$ (80\% neutrophils, $20 \%$ lymphocytes), a hemoglobin level of $8.5 \mathrm{~g} / \mathrm{dL}$, platelet count of $456,000 / \mathrm{mm}^{3}$, and C-reactive protein level of $9.2 \mathrm{mg} / \mathrm{dL}$. Serum electrolytes, renal function, and liver function were all normal. Cranial computed tomography revealed a brain abscess in the right temporal lobe. The patient was prescribed intravenous ceftriaxone $(100 \mathrm{mg} /$ $\mathrm{kg} /$ day in two doses), vancomycin $(60 \mathrm{mg} / \mathrm{kg} /$ day in four doses) and metronidazole (30 $\mathrm{mg} / \mathrm{kg} /$ day in three doses). Dexamethasone was also started for brain edema, and levetiracetam was started to control the seizures.

On the fourth day of treatment, the patient was referred to our clinic for evaluation by pediatric infectious disease specialists. On physical examination, he was conscious with symmetrical and equally reactive pupils and no meningeal sign or respiratory problem. He was agitated. There were accompanying exophthalmos, left facial paralysis, and purulent otorrhea. He had locomotor difficulties and could speak a few simple words with hearing loss. Hepatosplenomegaly was also detected. His height was 96 centimeters [-8.89 standard deviation (SD)], while his weight was 15 kilograms ( $-6.65 \mathrm{SD})$, respectively. Contrastenhanced magnetic resonance imaging of the brain showed a multiloculated lesion with perilesional edema and slight contrast enhancement in the right temporal lobe (approximately $6 \times 5 \times 4.5 \mathrm{~cm}$ in diameter); subfalcine herniation and uncal herniation were also seen (Figure 1 ). Post-intubation plain chest radiography showed diffuse

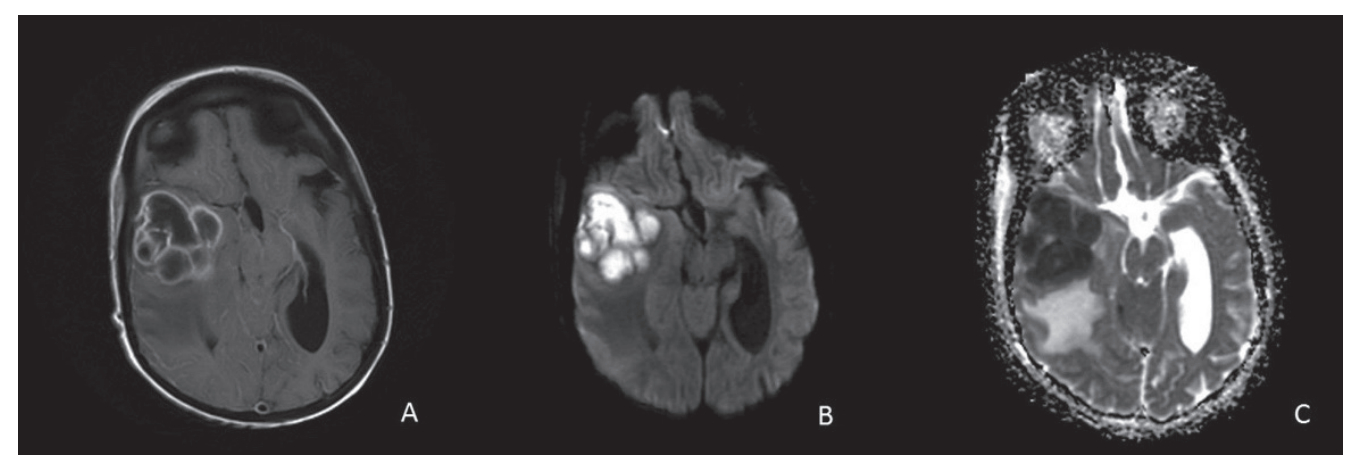

Figure 1. (A) Brain abscess with peripheral contrast enhancement in the right temporal lobe. (B, C) Reduced signal intensity on diffusion magnetic resonance images 
increased bone density and callus formation due to fracture healing (Figure 2).

The patient underwent abscess drainage via temporal-lobe burr-hole craniotomy under general anesthesia. Yellowishbrown pus was aspirated from the affected region and the abscess was excised completely. The abscess was grey-brownish, rigid, and filled with a necrotic material $(7 \times 5.8 \times 0.9 \mathrm{~cm}$ in diameter $)$. The abscess material was sent to the laboratory for microbiological and histopathological investigations. Gram staining showed gram-positive cocci and surgical drainage culture showed the growth of viridans streptococci, sensitive to $\beta$-lactam antibiotics. The histopathology report revealed active chronic inflammation, including abscess formation, proliferating blood vessels, congestion, and fibrosis, suggestive of pyogenic, intracerebral abscess formation. After surgery, the patient was followed in our pediatric intensive care unit (PICU) with respiratory support. He had an unfavorable clinical outcome, dying within 24 hours of admission to the PICU.

\section{Discussion}

The classification of osteopetrosis is challenging, due to variability in the severity of clinical manifestations, genetic factors and associated complications. Disease severity ranges from the occurrence of life-threatening complications in neonates to incidental findings of osteopetrotic features on plain radiography in adults with no complaint (8). Mutations in TNFRSF11A are associated with osteoclast-poor forms of autosomal recessive osteopetrosis because of impaired interaction between RANK (encoded by TNFRSF11A) with RANK ligand (encoded by TNFSF11)

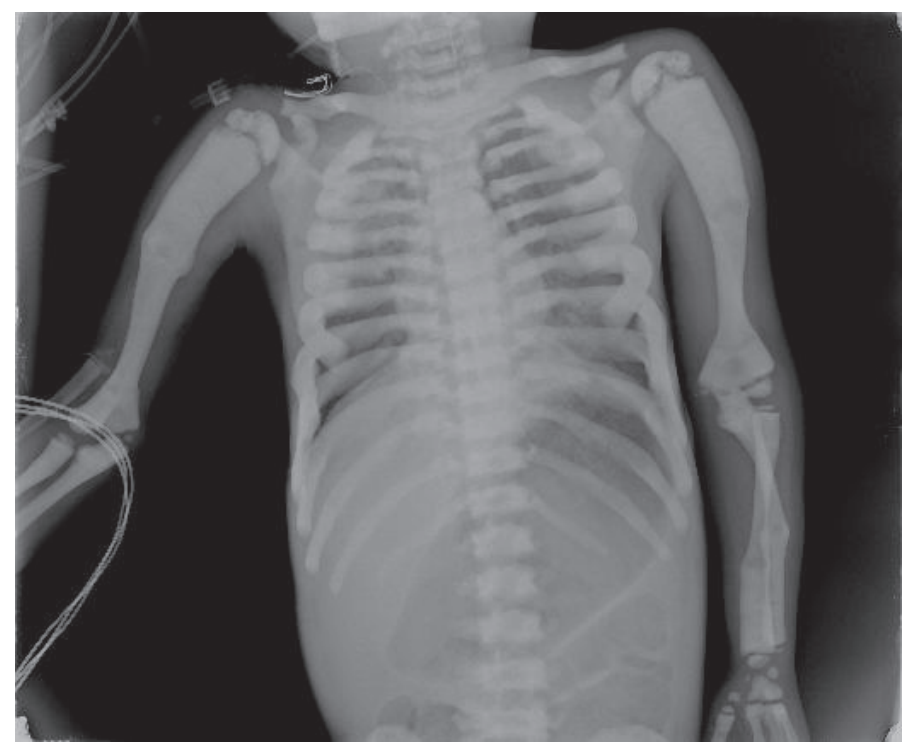

Figure 2. Diffuse increased bone density and formation of multiple calluses due to fracture healing which is important not only in osteoclast differentiation but also for immune system function $(2,3,7,9)$. Our patient had blindness caused by optic nerve compression, multiple fractures of the extremities and hypogammaglobulinemia. He was being followed by physicians from another center under a diagnosis of osteoclast-poor with immunoglobulin deficiency, autosomal recessive, infantile form OPTB7.

The most common infectious complication of osteopetrosis is osteomyelitis; in particular, mandibular osteomyelitis may be seen due to bone sclerosis and reduced vascular supply. Dental abscess formation and tooth decay are the main predisposing factors for infection $(8,10,11)$. Recurrent otitis media is another important and commonly seen entity $(10,11)$. Abnormal temporal bone anatomy, such as poor mastoid pneumatization and Eustachian tube narrowing, increases the risk of otologic infection (11).

Hypogammaglobulinemia due to bone marrow failure can also occur in patients with osteopetrosis and is an important risk factor for recurrent infection $(1,12,13)$. In one study, 15 of 32 patients with autosomal recessive osteopetrosis experienced multiple episodes of otitis media (11). Our patient had a history of recurrent, suppurative otitis media episodes in the past year, and physical examination revealed purulent otorrhea. The most common infections preceding brain abscess formation in children were sinusitis (36.3\%), periorbital/orbital cellulitis (16.1\%), otitis media $(13.5 \%)$ and meningitis (11.9\%) (14). Brain abscess formation should be suspected in patients with osteopetrosis, although only one case has been reported in the literature (6). This rare clinical entity can occur after antibiotherapy for otitis media episodes.

Brain abscess is aggressive and life-threatening infection with a high fatality rate, especially among immunocompromised children. Furthermore, a low GCS score at presentation is associated with a poor outcome (14). Our patient was immunodeficient and had a low GCS score at presentation. The clinical picture of brain abscess can be confusing and uncertain in the early phase of the disease. The initial symptoms are typically nonspecific, and few patients show the classic triad of headache, fever, and focal neurological deficits. In particular, brain abscess should be included in the differential diagnosis of immunocompromised patients with headache, altered mental status, vomiting, seizures, focal neurological deficits, and speech and visual disturbances (14).

The only effective treatment known for osteopetrosis is allogeneic bone marrow transplantation. Historically, the best outcomes have been achieved by using bone marrow from a genotypically human leukocyte antigen-identical 
donors $(2,3,15)$. Acute or chronic otitis media episodes are seen in half of all patients with osteopetrosis, the majority of whom require the insertion of tympanostomy tubes $(10,11)$. In our case, the patient had a history of chronic otitis media in the past year without appropriate treatment. The etiology of osteopetrosis is thought to be related to a contiguous focus of infection.

\section{Conclusion}

Therefore, a routine otologic examination should be an integral component of management and plays an important role in preventing more severe complications, such as brain abscess.

\section{Ethics}

Informed Consent: Informed consent was obtained from the patient for the publication of this case report and any accompanying images.

Peer-review: Externally peer-reviewed.

\section{Authorship Contributions}

Surgical and Medical Practices: Merve İşeri Nepesov, Eylem Kıral, Gürkan Bozan, Concept: Ömer Kılıç, Kürşat Bora Çarman, Coşkun Yarar, Ener Çağrı Dinleyici, Design: Merve İşeri Nepesov, Ömer Kılıç, Data Collection or Processing: Merve İşeri Nepesov, Ömer Kılıç, Suzan Şaylısoy, Analysis or Interpretation: Ömer Kılıç, Kürşat Bora Çarman, Coşkun Yarar, Suzan Şaylısoy, Ener Çağrı Dinleyici, Literature Search: Merve İşeri Nepesov, Ömer Kılıç, Writing: Merve İşeri Nepesov, Ömer Kılıç.

Financial Disclosure: The authors declared that this study received no financial support.

\section{References}

1. Sobacchi C, Schulz A, Coxon FP, Villa A, Helfrich MH. Osteopetrosis: genetics, treatment and new insights into osteoclast function. Nat Rev Endocrinol 2013;9:522-536. Epub 2013 Jul 23

2. Schulz A, Moushous D, Steward CG, Villa A, Sobacchi C. Osteopetrosis: consensus guidelines for diagnosis, therapy and follow-up. 2015. Last Accessed Date: 23.07.2020. Available from: https://esid.org/layout/set/ print/content/view/full/14267
3. Wu CC, Econs MJ, DiMeglio LA, Insogna KL, Levine MA, Orchard PJ, Miller WP, Petryk A, Rush ET, Shoback DM, Ward LM, Polgreen LE. Diagnosis and management of osteopetrosis: consensus guidelines from the Osteopetrosis Working Group. J Clin Endocrinol Metab 2017;102:3111-3123.

4. Infante-Cossio P, Gonzalez-Perez LM, Martinez-de-Fuentes $\mathrm{R}$, Infante-Cossio M, Castaño-Seiquer A, Jimenez-Castellanos E. Maxillomandibular osteomyelitis associated with osteopetrosis. J Craniofac Surg 2014;25:e79-e82.

5. Whyte MP, Wenkert D, McAlister WH, Novack DV, Nenninger AR, Zhang $\mathrm{X}$, Huskey M, Mumm S. Dysosteosclerosis presents as an "osteoclastpoor" form of osteopetrosis: comprehensive investigation of a 3-yearold girl and literature review. J Bone Miner Res 2010;25:2527-2539.

6. Srirompotong S, Saeng-Sa-Ard S, Srirompotong S. Otolaryngological complications of osteopetrosis. J Med Assoc Thai 2002;85:514-518.

7. Guerrini MM, Sobacchi C, Cassani B, Abinun M, Kilic SS, Pangrazio A, Moratto D, Mazzolari E, Clayton-Smith J, Orchard P, Coxon FP, Helfrich MH, Crockett JC, Mellis D, Vellodi A, Tezcan I, Notarangelo LD, Rogers MJ, Vezzoni P, Villa A, Frattini A. Human osteoclast-poor osteopetrosis with hypogammaglobulinemia due to TNFRSF11 A (RANK) mutations. Am J Hum Genet 2008;83:64-76.

8. Stark Z, Savarirayan R. Osteopetrosis. Orphanet J Rare Dis 2009;4:5.

9. Das S, Sepahi I, Duthie A, Clark S, Crockett JC. RANK receptor oligomerisation in the regulation of NFKB signalling. J Mol Endocrinol 2014;53:81-91. Epub 2014 May 23

10. Stocks RM, Wang WC, Thompson JW, Stocks MC 2nd, Horwitz EM. Malignant infantile osteopetrosis: otolaryngological complications and management. Arch Otolaryngol Head Neck Surg 1998;124:689-694.

11. Dozier TS, Duncan IM, Klein AJ, Lambert PR, Key LL Jr. Otologic manifestations of malignant osteopetrosis. Otol Neurotol 2005;26:762766.

12. El-Sobky TA, Elsobky E, Sadek I, Elsayed SM, Khattab MF. A case of infantile osteopetrosis: the radioclinical features with literature update. Bone Rep 2015;4:11-16.

13. Michou L, Brown JP. Genetics of bone diseases: Paget's disease, fibrous dysplasia, osteopetrosis, and osteogenesis imperfecta. Joint Bone Spine 2011;78:252-258. Epub 2010 Sep 19

14. Felsenstein S, Williams B, Shingadia D, Cox on L, Riordan A, Demetriades AK, Chandler CL, Bassi S, Koutoumanou E, Stapleton $\mathrm{S}$, Sharland M, Bryant PA. Clinical and microbiologic features guiding treatment recommendations for brain abscesses in children. Pediatr Infect Dis J 2013;32:129-135.

15. Orchard PJ, Fasth AL, Le Rademacher J, He W, Boelens JJ, Horwitz EM, Al-Seraihy A, Ayas M, Bonfim CM, Boulad F, Lund T, Buchbinder DK, Kapoor N, O'Brien TA, Perez MAD, Veys PA, Eapen M. Hematopoietic stem cell transplantation for infantile osteopetrosis. Blood 2015;126:270-276. Epub 2015 May 26 